\title{
Response to Jefford
}

\author{
Larissa Nekhlyudov, Lawrence N. Shulman, Paul B. Jacobsen, Michelle A. Mollica, \\ Ann M. Geiger, Deborah K. Mayer
}

See the Notes section for the full list of authors' affiliations.

Correspondence to: Larissa Nekhlyudov, MD, MPH, Brigham \& Women's Primary Care Associates, 800 Huntington Ave, 6th fl, Boston, MA 02115 (e-mail: lnekhlyudov@ partners.org).

We appreciate the letter by Dr Michael Jefford in response to our recent paper describing the quality of cancer survivorship framework. As he points out, we specifically developed it to provide "an essential reference, to build and assess survivorship care efforts." The issues he raises are relevant in delivering survivorship care using this framework. Dr Jefford brings up an important point regarding clinician burnout, which is becoming prevalent in the United States and internationally $(1,2)$. Solutions to this problem are being addressed by the National Academy of Medicine Action Collaborative on Clinician WellBeing and Resilience, a network of more than 60 organizations convened in 2017 (2). In cancer survivorship care, finding ways to reduce burden on health-care providers will need specific attention when developing and testing models of follow-up (3). Transitioning survivors from oncology specialists to survivorship clinics or primary care providers may leave them seeing more patients on active treatment or at the end of life and may potentially curb the "joy" of seeing long-term "success" survivors. However, failing to transition may lead to additional workload of caring for large panels of long-term survivors and thus increase stress and burnout. Caring for cancer survivors with complicated histories, treatment exposures, and chronic medical conditions may also endanger the well-being of primary care clinicians. The nursing workforce in all settings is being threatened (4). Our framework allows for flexible methods of assessing and monitoring the suggested domains and developing innovative approaches in doing so. It takes into consideration not only the patients and their needs, but also the providers and systems supporting survivorship care. Sustaining and increasing a workforce to meet the needs of the growing population of cancer survivors is critical, and organizations may consider adopting the ongoing calls to expand the Institute for Healthcare Improvement's "Triple Aim" to a "Quadruple Aim" that also seeks to improve the work life of health-care providers (5).

We fully support the widely accepted definition of cancer survivor as an individual from the time of diagnosis and through the balance of his or her life (6). Therefore we agree with Dr Jefford that the domains and indicators outlined in our model should be applied to patients before and during cancer treatment. As such, we encourage the assessment and monitoring of psychosocial and physical effects as well as chronic medical conditions and health promotion throughout the cancer care continuum. In measuring the quality of cancer survivorship care, we emphasize the need to focus greater attention on cross-cutting outcomes, or endpoints, that are applicable and comparable with other prevalent conditions such as cardiovascular disease. We believe our framework provides a blueprint for delivering quality cancer survivorship care and supports the six elements outlined by the Institute of Medicine for health care to be safe, effective, patientcentered, timely, efficient, and equitable (7).

\section{Notes}

Affiliations of authors: Department of Medicine, Brigham and Women's Hospital, Harvard Medical School, Boston, MA (LN); Abramson Cancer Center, University of Pennsylvania, Philadelphia, PA (LNS); Healthcare Delivery Research Program, National Cancer Institute, Bethesda, MD (PBJ, MAM, AMG); UNC Lineberger Comprehensive Cancer Center and School of Nursing, University of North Carolina-Chapel Hill, NC (DKM); Office of Cancer Survivorship, National Cancer Institute, Bethesda, MD (DKM).

The views expressed are those of the authors and do not necessarily represent the official views of the National Cancer Institute.

\section{References}

1. Medscape Global Physicians' Burnout and Lifestyle Comparisons 2019. https:// www.medscape.com/slideshow/2019-global-burnout-comparison-6011180. Accessed June 12, 2019.

2. National Academy of Medicine Action Collaborative on Clinician Well-Being and Resilience. https://nam.edu/initiatives/clinician-resilience-and-well-being. Accessed June 12, 2019.

3. Alfano CM, Jefford M, Maher J, Birken SA, Mayer DK. Building personalized cancer follow-up care pathways in the United States: lessons learned from 
implementation in England, Northern Ireland, and Australia. Am Soc Clin Oncol Educ Book. 2019;39:625-639.

4. Supply and Demand Projections of the Nursing Workforce: 2014 2030 July 21, 2017. https://bhw.hrsa.gov/sites/default/files/bhw/nchwa/ projections/NCHWA_HRSA_Nursing Report.pdf. Accessed June 12, 2019.

5. Bodenheimer T, Sinsky C. From triple to quadruple aim: care of the patient requires care of the provider. Ann Fam Med. 2014;12(6):573-576.
6. Office of Cancer Survivorship, Division of Cancer Control and Population Sciences, National Cancer Institute. https://cancercontrol.cancer.gov/ocs/statistics/definitions.html. Accessed June 12, 2019.

7. Committee on Quality Health Care in America, Institute of Medicine. Crossing the Quality Chasm: A New Health System for the 21st Century. Washington, DC: National Academy Press; 2001. 